\title{
Ortopedik cerrahi ve venöz tromboembolizm profilaksi ajanları: farmakolojik yöntemler
}

\author{
Orthopedic surgery and venous thromboembolism prophylaxis agents: \\ pharmacological methods
}

\author{
Hasan Hüseyin Ceylan ${ }^{1}$ İbrahim Azboy ${ }^{2}$ \\ ${ }^{1}$ Muş Devlet Hastanesi, Ortopedi ve Taravmatoloji Kliniği, Muş \\ ${ }^{2}$ İstanbul Medipol Üniversitesi, Tıp Fakültesi, Ortopedi ve Travmatoloji Anabilim Dalı, İstanbul
}

\begin{abstract}
Majör ortopedik cerrahiler, venöz tromboembolizm (VTE) gelişimi açısından risk teşkil eder. Hastanın ameliyat sonrası dönemde komplike olmaması için, tüm güncel kılavuzlar tarafından VTE proflaksi uygulaması önerilmektedir. Bu uygulamanın nasıl yapılacağı konusunda nihai bir fikir birliği olmamakla birlikte, farklı ajan ve rejim önerileri literatürde halen tartışılmaktadır. Bu derlemede, ortopedik cerrahi sonrası VTE proflaksisinde kullanılan ajanlar genel hatlarıyla ele alınmış; ilaçların etki mekanizmaları, kullanım şekilleri, doz ayarlamaları, olası toksisite durumları ve antidotları güncel literatür ışı̆̆ında incelenmiştir. İdeal proflaksi ajanı belirlenirken hastaya özgü karar verilmeli ve devamlı güncellenmekte olan literatür kontrol edilmelidir.
\end{abstract}

Anahtar sözcükler: venöz tromboembolizm; VTE; proflaksi
V enöz tromboemboli (VTE), majör ortopedik girişimler sonrası karşılaşılan önemli bir komplikasyondur. Literatürde, asemptomatik VTE görülme sıklığı yüksek oranda bildirilmektedir. Ancak, semptomatik VTE klinik olarak önem arzeder. Ortopedik cerrahi sonrası VTE profilaksisi uygulanmayan hastalarda, 35 gün içinde semptomatik VTE görülme riski \%4,3 civarındadır. ${ }^{[1]}$ Total eklem replasmanı sonrası profilaksi uygulanan hastalarda semptomatik ve asemptomatik pulmoner emboli (PE) gelişme riski, sırasıyla \%0,4 ve \%23 olarak bildirilmiştir. ${ }^{[2]}$ Primer artroplasti cerrahisi sonrası fatal PE gelişme riski, uygulanan kemoprofilaksi ajanından bağımsız olarak, \%0,1-0,2 olarak bildirilmektedir. ${ }^{[3]}$ Fatal olmayan VTE olgularında ise; hastanede yatış süresinin uzaması ve aylarca süren medikal tedavi, gerek hasta gerekse sağlık sistemi için ciddi yükleri beraberinde getirmektedir.

\begin{abstract}
Major orthopaedic surgeries have higher risk in terms of venous thromboembolism (VTE). All current guidelines recommend VTE prophylaxis to prevent postoperative complications. Although, there is not a concensus, there are multiple agents and regimes discussed in current literature. In this review, we aimed to discuss currently available VTE prophylaxis agents, in terms of mechanism, usage, dosing, possible toxicity, and available antidots. To have an optimum prophylaxis and before making a decision, surgeon should consider patient spesific factors, and update his/her literature knowledge.
\end{abstract}

Key words: venous thromboembolism; VTE; prophylaxis

Doku travması ya da damar endotelinin zedelenmesini takiben salınan doku faktörü, koagülasyon kaskadını tetikler. ${ }^{[4]} \mathrm{Bu}$ açıdan bakıldığında, tüm majör ortopedik cerrahiler VTE açısından riskli ameliyatlardır. Bu nedenle, majör ortopedik cerrahilerden sonra VTE riskini azaltmak amacıyla, yaklaşık 20 yıldır birçok kılavuzda farmakolojik profilaksi önerilmekte ve uygulanmaktadır. ${ }^{[1,5,6]}$ Ancak, uygun hastada doğru VTE ajanının tercih edilmesi önemlidir. Çünkü malignensi, pulmoner hipertansiyon, miyeloproliferatif hastalıklar gibi eşlik eden hastalıklar veya VTE öyküsünün olması durumunda, profilaksi ajanlarının yetersiz kullanılması VTE riskinde artışa neden olurken, kanama riski göz önünde bulundurulmaksızın potent antikoagülanların kullanılması da majör kanamalara yol açar. Bu nedenle son yıllarda, VTE profilaksi yöntemine karar verirken her hasta için risk analizi yapılması konusu gündeme gelmiştir. Nitekim, 2007[5]

- İletişim adresi: Doç. Dr. İbrahim Azboy, İstanbul Medipol Üniversitesi, Ortopedi ve Travmatoloji Anabilim Dalı, İstanbul, Türkiye Tel: 0216 - 5446666 / 6254 e-posta: ibrahimazboy@gmail.com

- Geliş tarihi: 22 Şubat 2019 Kabul tarihi: 6 Ağustos 2019 


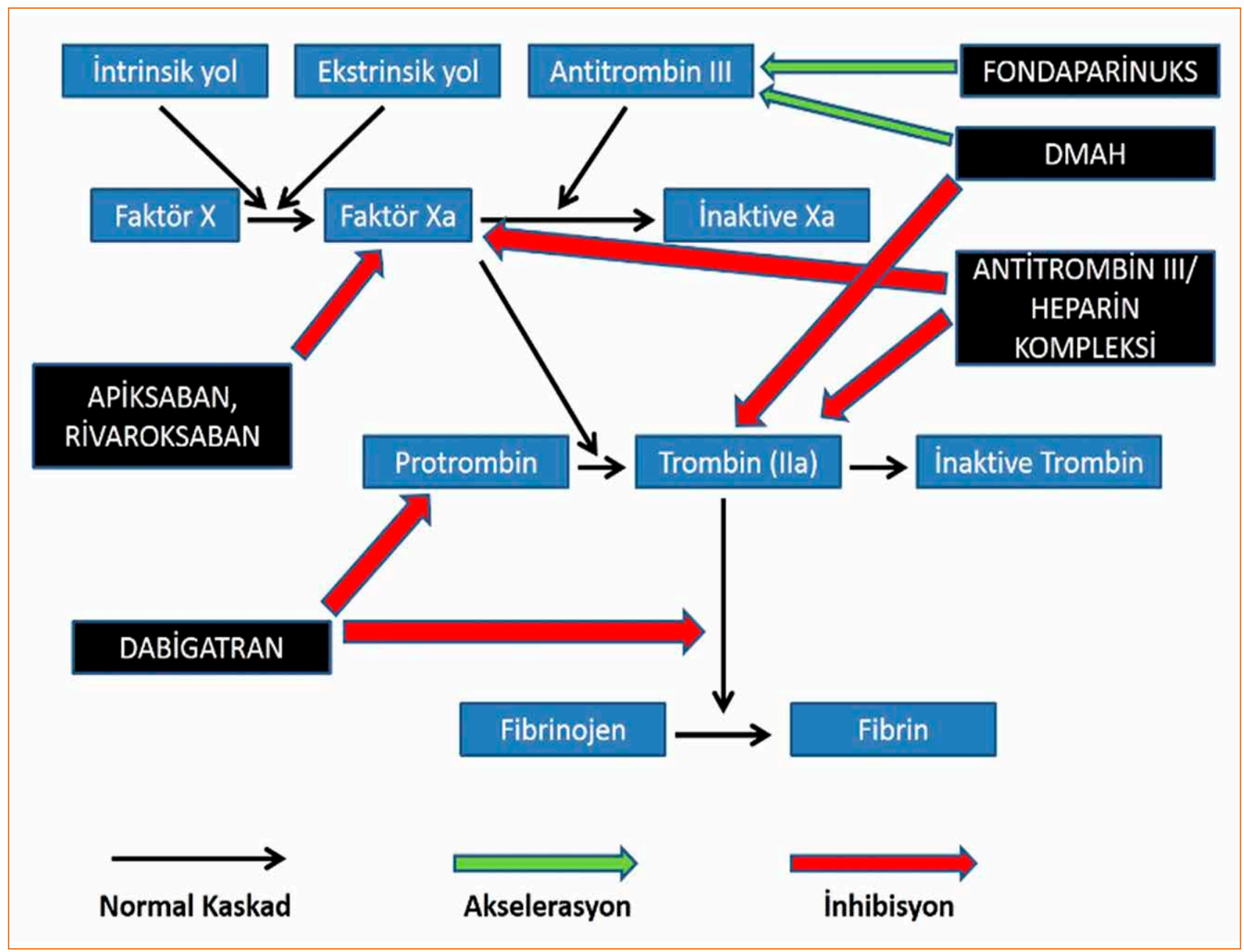

Şekil 1. Pıhtılaşma kaskadı ve antitrombotiklerin etki basamakları. ${ }^{[20]}$

ve $2011^{[7]}$ yıllarında yayımlanan American Academy of Orthopaedic Surgeons (AAOS) kılavuzları, VTE profilaksisinde kullanılacak ajana karar verirken risk analizi yapılmasını önermektedir. Bu amaca yönelik olarak hasta, VTE riski açısından olduğu kadar, kanama riski açısından da ayrıntılı olarak değerlendirildikten sonra en uygun profilaksi ajanı belirlenmelidir. ${ }^{[8]}$

Farmakolojik VTE profilaksisinde kullanılan ajanlar, pıhtılaşma kaskadının farklı noktalarına etki ederek VTE oluşumunu engellemeyi amaçlar (Şekil 1). Bu ajanların farmakolojik özellikleri arasında farklar vardır. Dolayısıyla, uygun ajanı tercih edebilmek, doğru uygulamak ve uygulama sırasında oluşabilecek yan tesirleri yönetebilmek için, bu ajanlar hakkında ayrıntılı bilgi sahibi olmak gerekir. Bu derlemede, ortopedik cerrahi sonrası VTE profilaksisinde kullanılan farmakolojik ajanların tipleri, etki mekanizmaları, başlanma zamanları, uygulama yolları, antidotları ve yan tesirleri hakkında genel bilgi verilmesi amaçlanmıştır.

Ortopedik cerrahi sonrası VTE profilaksisinde kullanılan ajanlar, genel olarak yedi grupta sınıflandırılabilir (Tablo 1):

a) Vitamin $\mathrm{K}$ antagonistleri

b) Heparin

c) Düşük molekül ağırlıkı heparinler (DMAH)

d) İndirekt Faktör Xa inhibitörleri

e) Direkt Faktör Xa inhibitörleri

f) Direkt trombin inhibitörleri

g) Asetilsalisilik asit 
Tablo 1. VTE profilaksisinde sık kullanılan ajanlar, etki mekanizmaları, önerilen ilaç başlanma zamanlaması, monitorizasyon yöntemleri ve antidotları

\begin{tabular}{|c|c|c|c|c|c|}
\hline Grup & Etken madde & Etki Mekanizması & Başlanma Zamanı & Monitorizasyon & Özel Antidot \\
\hline $\begin{array}{l}\text { Vitamin } \mathrm{K} \\
\text { antagonistleri }\end{array}$ & Warfarin & $\begin{array}{l}\text { Faktör II, VII, IX ve X } \\
\text { sentezinin inhibisyonu }\end{array}$ & Cerrahiden $12-24$ saat sonra ${ }^{a}$ & INR & $\begin{array}{l}\text { Vitamin K, } \\
\text { taze donmuş } \\
\text { plazma, } \\
\text { konsantre } \\
\text { protrombin } \\
\text { kompleksi }\end{array}$ \\
\hline UFH & Heparin & Antitrombin III aktivasyonu & Cerrahiden 6-12 saat sonrab & aPTT & Protamin sülfat \\
\hline $\begin{array}{l}\text { Düşük moleküler } \\
\text { ağırlıklı heparin }\end{array}$ & $\begin{array}{l}\text { Enoksoparin } \\
\text { Bemiparin } \\
\text { Dalteparin } \\
\text { Tinzaparin } \\
\text { Parnaparin }\end{array}$ & $\begin{array}{l}\text { AT III üzerinden } \\
\text { Faktör Xa inhibisyonu }\end{array}$ & Cerrahiden $12-24$ saat sonra ${ }^{b}$ & Antifaktör Xa & $\begin{array}{l}\text { Protamin sülfat, } \\
\text { Antifaktör Xa }\end{array}$ \\
\hline $\begin{array}{l}\text { Indirekt Faktör Xa } \\
\text { inhibitörleri }\end{array}$ & Fondaparinuks & $\begin{array}{l}\text { AT III'e bağlanıp yapısal } \\
\text { değişikliğe neden olarak } \\
\text { Faktör Xa'nın indirekt } \\
\text { inhibisyonu }\end{array}$ & Cerrahiden 6-12 saat sonrab & Antifaktör Xa & $\begin{array}{l}\text { Rekombinant } \\
\text { aktive Faktör VII }\end{array}$ \\
\hline $\begin{array}{l}\text { Direkt Faktör Xa } \\
\text { inhibitörleri }\end{array}$ & $\begin{array}{l}\text { Apiksaban } \\
\text { Rivaroksaban }\end{array}$ & $\begin{array}{l}\text { Faktör Xa üzerinde selektif } \\
\text { inhibisyon }\end{array}$ & 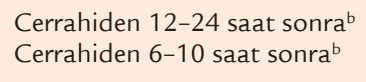 & $\begin{array}{l}\text { Yok } \\
\text { Yok }\end{array}$ & $\begin{array}{l}\text { Yok } \\
\text { Yok }\end{array}$ \\
\hline $\begin{array}{l}\text { Direkt trombin } \\
\text { inhibitörleri }\end{array}$ & Dabigatran & $\begin{array}{l}\text { Trombin'in direkt } \\
\text { inhibisyonu }\end{array}$ & Cerrahiden $1-4$ saat sonrab & Trombin Zamanı & Idaricuzimab \\
\hline Asetilsalisilik asit & Asetilsalisilik asit & $\begin{array}{l}\text { Trombosit agregasyonunun } \\
\text { inhibisyonu, Faktör } \\
\text { V inhibisyonu ile } \\
\text { protrombinaz üretimini } \\
\text { azaltır, Trombin } \\
\text { üretimini zayıflatır, } \\
\text { COX-1 inhibisyonu ile } \\
\text { TXA }_{2} \text { üretimini azaltarak } \\
\text { vazodilatasyon }\end{array}$ & Cerrahiden $6-12$ saat sonra ${ }^{b}$ & Kanama zamanı & Yok \\
\hline
\end{tabular}

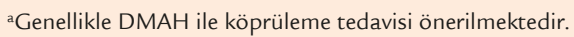

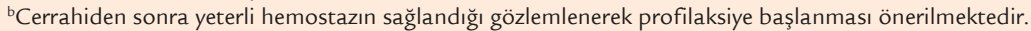

\section{Vitamin K Antagonistleri}

Warfarin sodyum etken maddesi içeren (Türkiye'deki ticari isimler: Coumadin ${ }^{\circledR}$, Orfarin $\left.^{\circledR}\right)$ ilaçlardır. Warfarin, hızlı şekilde etki gösteren oral bir antikoagülan ajandır; kalsiyuma bağlı pıhtılaşma faktörlerinin (Faktör II, VII, IX ve X) biyolojik aktif formlarının ve regülatuvar protein $C$, $S$ ve Z'nin sentezinin inhibisyonu yoluyla etki eder. ${ }^{[4,9,10]}$ Terapötik aralığı çok dar olan bu ilacın günlük dozu, oral olarak kullanılan 5-10 mg'dır. Warfarinin etkisi "international normalized ratio" (INR) ile takip edilir. INR, protrombin zamanının (PZ) standardize edilmiş oranıdır. ${ }^{[11]}$ Sitokrom P450 sistemi ile metabolize olan warfarinin kan değerleri, aynı metabolizmayla atılan diğer ilaç ve besinlerden etkilenmekte ve bu durum INR değerlerinin kontrolünü zorlaştırabilmektedir. ${ }^{[12]}$ Warfarin vücuttan esas olarak böbreklerden atılır. ${ }^{[13]}$ Warfarin kullanımında karşılaşılabilen en ciddi yan etki kanamadır. Özellikle INR değeri 5'in üzerinde ise bu risk daha ciddi oranda artar. ${ }^{[12]}$ ileri yaş, eşlik eden hastalıklar, ilave ilaçların kullanımı gibi hallerde bu yan etkinin kontrolü daha zorlaşır. ${ }^{[12]}$ Olası bir kanama durumunda, antidot olarak $\mathrm{K}$ vitamini, taze donmuş plazma (TDP) ya da konsantre protrombin kompleksi uygulanabilir. ${ }^{[14,15]}$ Tüm ameliyat sonrası süreçte warfarin kullanan merkezler olduğu gibi, erken dönemde warfarin kullanıp taburculukta DMAH'ye geçen merkezler de vardır. Warfarin, elektif total kalça artroplastisi (TKA) ve total diz artroplastisi (TDA) sonrası VTE profilaksisinde etkinliği kabul görmüş ve uzun süredir kullanılmakta olan bir ajan olmasına rağmen, ameliyat sonrası erken dönemde antikoagülan etkinliğinin yeterli olmaması, sık INR takibi gerektirmesi ve kanama riski nedeniyle, son yıllarda kullanımı giderek azalmıştır. ${ }^{[16]}$ 


\section{Heparin}

Heparin sodyum (Türkiye'deki ticari isimler: Heparin Sodyum ${ }^{\circledR}$, Heparinum ${ }^{\circledR}$, Koparin ${ }^{\circledR}$, Nevparin ${ }^{\circledR}$, Poliparin $^{\circledR}$, Seloparin ${ }^{\circledR}, \operatorname{Vasparin}^{\circledR}$ ) etken maddesi içeren ilaçlar bu gruba girer. Standart heparin, literatürde genellikle unfraksiyone heparin (UFH) olarak adlandırılır. Heparin doğal olarak vücudumuzda mast hücreleri tarafindan da üretilen pıhtılaşma engelleyici bir proteoglikandır. Heparinler, Antitrombin III'e (AT III) bağlanarak onun etkisini arttıran, indirekt yolla etki gösteren antikoagülanlardır. Etkinliği artan AT III ise, Faktör Xa ve Faktör Ila inhibisyonuna neden olur ve nihayetinde trombin aktivasyonunu engeller. ${ }^{[17]}$ Akut VTE olaylarının tedavisinde sıklıkla tercih edilen UFH, intravenöz ya da subkutan olarak uygulanabilir. İntravenöz uygulama hemen etkisini gösterirken, subkutan uygulamada terapötik etki 20-60 dk içinde başlar. ${ }^{[11]}$ illaç iki yolla vücuttan atılır. Bir kısmı endotelial düzeyde depolimerize edilir ve bir kısmı da böbreklerden atılır. Fakat, tedavi dozundaki UFH uygulamasında asıl yıkım birinci yolla olduğundan, böbrek fonksiyonlarının takibi gerekmez. ${ }^{11]}$ Uygulama sonrası monitorizasyonda, altı saatte bir aktive parsiyel tromboplastin zamanı (aPTT) kontrolü önerilir. Tedavi dozu belirlenince bu aralık uzatılabilir. ${ }^{[18]}$ Diğer antikoagülan ajanlarda olduğu gibi, istenmeyen ve en sık görülen yan etki kanamadır. Diğer iyi bilinen bir yan etki ise, heparin kaynaklı trombositopenidir (Heparin Induced Thrombocytopenia -HIT). Olası HIT durumunda UFH'nin kesilmesi ve direkt trombin inhibitörü ya da Faktör Xa inhibitörü ile tedaviye devam edilmesi önerilir. ${ }^{[9]}$ Heparinin antidotu protamin sülfattır. Kısa yarılanma ömrü nedeniyle, toksisite kontrolü genelde kolaydır.

\section{Düşülk Molekül Ağırlıklı Heparinler (DMAH)}

Enoksaparin (Türkiye'deki ticari isimler: Axeparin ${ }^{\circledR}$, Clexane $^{\circledR}$, Enox $^{\circledR}$, Oxapar $^{\circledR}$ ), bemiparin (Türkiye'deki ticari ismi: Hibor ${ }^{\circledR}$ ), dalteparin (Türkiye'deki ticari isim: Fragmin ${ }^{\circledR}$ ), tinzaparin (Türkiye'deki ticari isim: Innohep ${ }^{\circledR}$ ) ve parnaparin (Türkiye'deki ticari isim: Fluxum $^{\circledR}$ ) etken maddesi içeren ilaçlar DMAH grubundadır. Bu moleküller, farklı yapı ve farmakolojik özellikleri nedeniyle birbirlerine ya da heparine dönüşemezler. ${ }^{[19]}$ DMAH'ler heparin grubundandır ve AT III'e bağlanarak onun antikoagülan etkisini arttırır. ${ }^{[17,20]}$ UFH'e göre daha küçük molekül yapısına sahip olan DMAH'lerin AT III üzerinden Faktör Xa inhibisyonunun Faktör Ila'dan oransal olarak daha iyi olduğu bilinmektedir. ${ }^{[17,20]}$ Subkutan uygulama sonrası 3-5 saatte, dolaşımda pik doza ulaşılır ve bu moleküllerin yarılanma ömrü UFH'ye göre daha uzundur (17-21 saat). ${ }^{[10]}$ UFH'ye kıyasla doz-etki profili daha öngörülebilir olan DMAH grubu, VTE profilaksisi için sık kullanılan ajanlar arasındadır. ${ }^{[20,21]} \mathrm{DMAH}$ uygulaması sonrasında, warfarin ya da UFH'de olduğu gibi, sıkı laboratuvar takibi gerekmez. ${ }^{[9]}$ Tedavi dozunda DMAH kullanan hastalarda, cerrahiden 12 veya 24 saat önce ilacın kesilmesi, ameliyat sonrası 6-12 saat sonra başlanması önerilir. ${ }^{[22]}$ ihtiyaç halinde, monitorizasyon için Antifaktör Xa seviyesi ile kontrol yapılabilir. ${ }^{[20]}$ Monitörizasyon gerektirmemesi ve sabit dozda etkili profilaksi sağlamasına karşın, cilt altı uygulama gerekliliği, pahalı olması, yara yeri akıntısında uzama, kanama ve bu nedenle artan enfeksiyon riski, DMAH'lerin dezavantajları arasında sayılır. ${ }^{[23]}$ ihtiyaç halinde antidot olarak kullanılan protamin sülfat, DMAH'lerin antikoagülan etkisini \%60 oranında geri çevirebilir. ${ }^{[24]}$ Antifaktör Xa, bir diğer antidottur. Ciddi kanama olgularında kriyopresipitat ve taze donmuş plazma uygulaması ise diğer tedavi metodlarıdır. ${ }^{[20]}$ Beş-yedi gün DMAH kullanımı sonrası da HIT gelişebilir. Trombosit sayısında \%50'ye varan düşüşle kendini gösteren bu durumda, DMAH'lerin kesilmesi ve direkt trombin inhibitörü ya da Faktör Xa inhibitörü ile tedaviye devam edilmesi önerilir. ${ }^{\left[{ }^{9]}\right.}$ Olası HIT tablosunun atlanmaması için, DMAH ugulamasının birinci haftasında tam kan sayımı ile trombosit sayısının kontrolü önerilir. Uzun dönem kullanım sonrası, UFH kadar olmasa da, DMAH nedenli osteoporoz bildirilmiştir. ${ }^{10]}$ DMAH'ler esas olarak böbreklerden atılmaktadır. Bu nedenle, böbrek yetmezliği hastalarında ilacın yarılanma ömrü uzayacağı için doz ayarlaması önerilir. [25]

\section{İndirekt Faktör Xa İnhibitörleri}

Fondaparinuks, sodyum etken maddesi içeren (Türkiye'deki ticari isim: Arixtra ${ }^{\circledR}$ ) bir ilaçtır; heparinlerde bulunan doğal pentasakkaritin sentetik analoğudur. İndirekt Xa inhibitörleri AT III'e direkt bağlanarak, yapısal değişikliğe neden olup Faktör Xa'yı indirekt şekilde inhibe eder. ${ }^{[20]}$ Fondaparinuks, trombin üzerinde hiçbir etkisi olmadan antitrombine bağlanarak etki gösterir. Plateletlerle ya da platelet Faktör IV ile etkileşime girmez ve bu nedenle HIT tablosuna neden olmaz. ${ }^{[26]}$ Fondaparinuks selektif ve irreversibl olarak AT III'e bağlanır ve esas olarak böbreklerden atılır. Böbrek yetmezliği olan hastalarında bu ajanın kullanımı kontraendike olarak kabul edilmektedir. ${ }^{[9]}$ Aynı zamanda, $50 \mathrm{~kg}$ altında olan bireylerde de bu ilacın kullanılması önerilmez. Subkutan uygulama sonrası hızla ve tamamen absorbe olur ve böbrek fonksiyonları normal olan bir bireyde yarı ömrü 17-21 saattir. ${ }^{[27]}$ Yetmiş beş yaş üstü hastalarda böbreklerden atılımı görece yavaş olabilmektedir. Bu nedenle, 
başlangıç dozu günlük $75 \mathrm{mg}$, devam dozu $150 \mathrm{mg}$ olarak önerilir. ${ }^{[28,29]}$ Fondaparinuks kullanımı sırasında, rutin olarak Faktör Xa ile monitörizasyon gerekmez. Fondaparinuks kullanımına bağlı majör kanama riskinin DMAH'lere göre istatistiksel olarak daha sık olduğu bildirilmiştir. ${ }^{\left[{ }^{30]}\right.}$ Fondaparinuks uygulamasına ameliyattan altı saat sonra başlanması önerilir. ${ }^{[31,32]}$ Uzun yarılanma ömrü nedeniyle, olası toksisite tablosu ciddi sorun olabilir. Antidot olarak rekombinant aktive Faktör VII (rVIla) ile koagülasyon ve hemostaz sağlanabileceği bildirilmiştir. ${ }^{[33]}$

\section{Direkt Faktör Xa İnhibitörleri}

Bu grupta, apiksaban (Türkiye'deki ticari isim: Eliquis ${ }^{\circledR}$ ), rivaroksaban (Türkiye'deki ticari isim: Xarelto $^{\circledR}$ ) ve edoksaban (Türkiye'deki ticari isim: Lixiana ${ }^{\circledR}$ ) gibi ajanlar bulunur. Bu ajanlar, tromboz kaskadındaki Faktör Xa'yı selektif olarak inhibe ederek antikoagülan etki sağlamaktadır. ${ }^{[10]} \mathrm{Bu}$ ajanlar ayrıca, protrombinaz aktivitesini de inhibe eder. ${ }^{[34]}$ Heparin kullanımı komplikasyonu olan HIT durumunda da bu ilaçların kullanımı önerilir. ${ }^{[9]}$

Rivaroksaban, üç saat içinde kanda pik yaparak hızla etki gösteren bir moleküldür. Oral alım sonrası biyoyararlanım \%60-80 civarındadır. CYP3A4 tarafindan metabolize edilir, hem böbreklerden hem de fekal/safra yoluyla vücuttan atılır. Yarılanma ömrü 5-9 saattir. ${ }^{[35]}$ Günde tek doz kullanılan rivaroksabanın, farmakolojik etkisi 24 saat devam eder. Farmakokinetik olarak öngörülebilir bir davranış sergilediği için, monitorizasyona ihtiyaç duyulmaz. ${ }^{[35]}$ Günlük doz, aç ya da tok şekilde oral olarak alınan $10 \mathrm{mg}$ şeklindedir. Cerrahi sonrası hemostazın sağlanması açısından, ameliyatı takip eden 6-10 saat içinde alınması önerilir. ${ }^{[35]}$

Apiksaban, oral olarak alınabilen, üç saat içinde maksimum kan değerine ulaşabilen, \%50-85 dolaylarında biyoyararlanımı olan, küçük molekül ağılıkIı bir ajandır. Daha çok safra yoluyla vücuttan atılır. Cerrahiden 12-24 saat sonra başlanmak üzere, günde iki defa 2,5 mg şeklinde kullanılması önerilir. Net olmamakla birlikte, böbrek yetmezliği olan hastalarda güvenli bir ajan olduğu bildirilmektedir. ${ }^{[29]}$

Direkt Faktör Xa inhibitörlerinin monitörizasyonu için belirlenen özel bir laboratuvar parametresi bulunmamaktadır. İlaç uygulamasını takip eden 1-4 saat sonra, protrombin zamanı (PT) ve aPTT'de uzama görülebilir. Fakat bu değişiklik hızla azalan ilaç tepe plazma seviyesine bağlı olduğundan, terapötik dozlarda PT, aPTT ve kanama zamanı değerleri çok değişmez. ${ }^{[11]}$ Tedavi dozunun aşıldığı durumlarda, doza bağımlı şekilde PT değerinde artış görülür. ${ }^{[11]}$ Esasında Antifaktör Xa seviyeleri, DMAH'lerin takibinde kullanılmak üzere tasarlanmış ve kalibre edilmiştir. Fakat,
Faktör Xa inhibitörlerinin aşırı dozunun tespitinde de Antifaktör Xa seviyesinin kontrolü önerilir. ${ }^{[11]}$ Tüm antikoagülan ilaçlarda olduğu gibi, direkt Xa inhibitörlerinin majör yan etkisi de kanamadır. Mekanizması tam olarak açıklanmamış olmakla birlikte, trombositopeni olguları da bildirilmiştir. ${ }^{[20]}$ Faktör Xa inhibitörlerinin özel bir antidotu yoktur, ancak Faktör VIla ve konsantre protrombin kompleksi kullanımını önerilir. ${ }^{[11,24]}$ Kuzey Amerika Tromboz ve Hemostaz Derneği, dört faktörlü konsantre protrombin kompleksi kullanımının en iyi yol olduğu görüşündedir. ${ }^{[36]}$

\section{Direkt Trombin İnhibitörleri (DTI)}

Dabigatran (Türkiye'deki ticari isim: $\operatorname{Pradaxa}^{\circledR}$ ) trombini direkt inhibe ederek etki gösteren bir direkt trombin inhibitörüdür (DTI). Aynı gruptaki diğer moleküller olan melagatran ve ksimelagatran, ciddi karaciğer hasarı yaptığı tespit edildiğinden, piyasadan çekilmiştir. ${ }^{[37]}$ Dabigatran oral, argatroban ve bivalirudin ise parenteral olarak uygulanabilen direkt trombin inhibitörleridir. Bunların heparinden farkı da bir faktöre ihtiyaç duymadan direkt trombini inhibe etmesidir. ${ }^{[12]}$ Bu ajanlar HIT olgularında da tercih edilebilir.

DTI laboratuvar monitörizasyonunda trombin zamanı ve ecarin pıhtılaşma zamanı (EPZ) testi kullanılır. ${ }^{[38]}$ Fakat, bu testler her laboratuvarda bulunmayan özellikli kitlerdir ve kullanımları çok kısıtlıdır. ${ }^{[38]}$ DTı monitörizasyonunda aPTT de kullanılabilir. Fakat aPTT değeri, terapötik dozun üzerine çıktığı bir noktadan sonra plato çizmeye başlar. Bu nedenle aPTT, DTI'lerinin doz aşımını belirlemede güvenilir değildir. ${ }^{[38]}$ Dabigatran, \%80 oranında böbreklerden atılır. Dolayısıyla, böbrek yetmezliği olan hastalarda dikkatle kullanılmalıdır. DTI kullanımı sonrası en sık karşılaşılan komplikasyonlar, gastrointestinal ve intrakraniyal kanamalardır. Bu komplikasyon doza bağlıdır ve 75 yaş üstü hastalarda daha sık rastlanır. ${ }^{[39]}$ Dabigatran antidotu olarak geliştirilen idarucizumabın, dakikalar içinde etki göstererek ilaç etkisini nötralize ettiği bildirilmiştir. ${ }^{[40]}$ Toksisite durumlarında tam kan ve TDP replasmanı önerilir. ${ }^{[41]}$ Böbrek fonksiyonu bozuk hastalarda koagülopati ve hayatı tehdit eden kanamaya neden olan dabigatran toksisitesi varsa, hemodiyaliz önerilir. ${ }^{[38]}$

\section{Asetilsalisilik asit}

Asetilsalisilik asit etken maddesi içeren (Türkiye'deki ticari isimler: Aspirin ${ }^{\circledR}, \operatorname{Ataspin}^{\circledR}$, Coraspin $^{\circledR}$, Dispri ${ }^{\circledR}$, Ecopirin $^{\circledR}$, Pharmaspirin ${ }^{\circledR}$ ) bir ilaçtır. Asetilsalisilik asit, antikoagülan, analjezik, antipiretik ve anti-inflamatuvar etkileri çok iyi bilinen ve 100 yıldan uzun süredir kullanılan bir moleküldür. Asetilsalisilik asidin, hem arteriyel hem venöz trombüs oluşumunu önlemede etkin olduğu çok sayıda çalışma ile gösterilmiştir. ${ }^{[42-45]}$ 
Asetilsalisilik asit, trombüs oluşunu önlemede çok sayıda mekanizma üzerinden etkili olmaktadır. Bilindiği gibi, trombüs oluşumunda trombositler önemli rol oynar. Asetilsalisilik asit ise, trombositleri inhibe ederek trombüs oluşumunu önlemede etkilidir. Asetilsalisilik asit, siklooksijenaz (COX) kanallarındaki serin rezidüsünün asetilasyonunu etkileyerek, megakaryositin katalitik bölgesine substrat akışını ve yeni üretilen trombositlerin agregasyon özelliğini engeller. Bu etki, yeni üretilen trombositin tüm hayatı boyunca sürer. ${ }^{[43,46]}$ Asetilsalisilik asit, özellikle COX-1 inhibisyon etkisi ile tromboksan $\mathrm{A}_{2}\left(\mathrm{TXA}_{2}\right)$ üretiminin azaltılması yoluyla vazodilatasyon sağlayarak da trombozu engellemektedir. ${ }^{[47]}$ Asetilsalisilik asidin VTE profilaksisindeki antitrombotik etkisi COX-1 inhibisyonu ile sınırlı değildir ve birçok diğer mekanizma da söz konusudur: NonCOX-1 yoluyla plateletlerin aktivasyon ve agregasyonunu baskılar. ${ }^{[48]}$ Antitrombin III ve protrombin asetilasyonu ile trombin üretimini azaltır ${ }^{[49,50]}$, monosit ve makrofajlardan doku faktörü salınımını azaltarak Faktör $V$ aktivasyonunu azaltıp protrombinaz formasyonunu bozar. ${ }^{[44,51]}$ Asetilsalisilik asit, fibrinojen ve fibrini asetilleyerek, trombin aracılı Faktör XII'nin aktivasyonunu engellemekte, sonuçta daha zayıf ve kolay yıkılabilir fibrin bağlantıları oluşmasına neden olmaktadır. ${ }^{[52,53]}$

Oral alım sonrası asetilsalisilik asit, mide ve barsaktan hızlı şekilde absorbe olup 40 dakika içinde maksimum plazma seviyesine çıkmakta ve bir saat içinde antiplatelet etkisini gösterir. ${ }^{[54]}$ Asetilsalisilik asit kullanımı sırasında toksisite durumu sık görülmez ve asetilsalisilik aside özel antidot yoktur. Olası toksisite durumunda, destek tedavisi ve kontrol edilemeyen durumlarda, hemodiyaliz önerilir.

Asetilsalisilik asidin diğer potent antikoagülanlara göre daha az kanamaya neden olması, ucuz olması, oral olarak kullanılabilmesi gibi avantajları vardır. ${ }^{[23]}$ 2007'de yayımlanan AAOS kılavuzu, asemptomatik VTE'den ziyade semptomatik VTE'nin engellenmesi konusuna odaklanmıştır. ${ }^{[5]}$ Bu kılavuzda; 1) PE ve majör kanama açısından standart riskli, 2) PE açısından standart / majör kanama açısından yüksek riskli ve 3) PE ve majör kanama açısından yüksek riskli hasta gruplarında; ayrı ayrı her üçü için de altı hafta boyunca günde iki defa $325 \mathrm{mg}$ asetilsalisilik asit kullanımını önerilmiştir. AAOS kılavuzundaki bu öneriye karşın, asetilsalisilik asit kullanımı için ideal doz ve süre konusunda halen görüş birliği bulunmamaktadır. ${ }^{[55]}$ COX-1 fonksiyonunun bloke edilmesi için günlük 75-150 mg aspirinin yeterli olduğu gösterilmiştir. ${ }^{[46]}$ Nitekim aspirinin, günde iki doz $81 \mathrm{mg}$ olarak kullanıldığında da günde iki doz 325 mg ile benzer etkinlik gösterdiği bildirilmiştir. ${ }^{[56]}$
2012 yılından önce yayımlanan ACCP kılavuzlarını hazırlayan panelistlerin ilaç firmaları olan ilişkileri belirlenmiştir. ${ }^{[57]}$ Bunun üzerine, 9. ACCP kılavuzu hazırlanırken, ilaç firmaları ile ilişkisi olan panelistler kılavuzların oylanması aşamalarından çıkarılmıştır. Bu düzenleme sonrasında, 2012'de yayımlanan 9. ACCP kılavuzunda $^{[1]}$ asetilsalisilik asit VTE profilakisinde güçlü bir öneri olarak yerini almıştır. En son olarak 2018 yılında yayımlanan NICE kılavuzu [6] VTE profilaksisinde asetilsalisilik asit kullanımını önermiştir.

\section{HANGI AJAN NE ZAMAN TERCIH EDILMELI? LITERATÜR NE DIYOR?}

Majör ortopedik cerrahi sonrası VTE profilaksisinin gerekliliği konusunda konsensus olmasına rağmen, optimum ajan konusunda literatürdeki tartışmalar halen devam etmektedir. Ortopedik cerrahların ameliyat sonrası VTE profilaksisinde kullanacakları ajana karar verirlerken, kanama riski, yara drenajı, kullanım kolaylığı ve etkinlik olarak dört temel kritere göre karar verdikleri tespit edilmiştir. ${ }^{[58]}$ American College of Chest Physicians (ACCP) tarafından 2012'de yayımlanan 9. kılavuzda, artroplasti sonrası profilakside; DMAH, fondaparinuks, dabigatran, apiksaban, rivaroksaban, düşük doz UFH, vitamin $\mathrm{K}$ antagonistleri ve asetilsalisilik asit önermektedir. ${ }^{[1]}$ AAOS 2007 ve 2011 kılavuzları da, kanama ve VTE dengesinin gözetilerek uygun profilaksiye karar verilmesini önermiştir. ${ }^{[21]}$

VTE profilaksisi literatürü, daha çok kalça ve diz artroplastisi sonrası yapılan çalışmalar üzerinden gelişmektedir. Ancak, bu literatürü değerlendirirken çaIsşmaların finasmanı konusunu da göz önünde bulundurmak önemlidir. Yakın zamanda yapılan bir sistematik derlemede, endüstri desteği ile yapılan çalışmalarda endüstri desteği olmayan çalışmalara göre, majör kanama, mortalite ve PE'nin daha az bildirildiği tespit edilmiştir. ${ }^{[59]} \mathrm{Bu}$ nedenle, literatür değerlendirilirken, çalışmaların endüstri desteği ile yapılıp yapılmadığına dikkat edilmesi yararlı olacaktır.

Amerikalı kalça ve diz cerrahlarının VTE profilaksi tercihlerini sorgulayan bir güncel çalışmada, ortopedi hekimlerinin \%68'inin ACCP yerine AAOS kılavuzunu esas aldığı tespit edilmiştir. ${ }^{[58]}$ Bu çalışmaya katılanlar, asetilsalisilik asidin kolay kullanım ve düşük kanama riski ile daha tercih edilen bir ajan olduğu kanaatindedirler. ABD ve İngiltere'de sıklıkla DMAH ve asetilsalisilik asit tercih edilmektedir. ${ }^{[60]} 2016$ 'da yapılan American Association of Hip and Knee Surgeons (AAHKS) kongresinde, 1200 üzerinde katılımcı ile gerçekleştirilen bir anket, ortopedistlerin \%80'den fazlasının kalça ve diz artroplastisi sonrası primer profilaksi ajanı olarak asetilsalisilik asidi tercih ettiğini ortaya koymuştur. ${ }^{[55]}$ 
Ülkemizde yeni yapılan bir çalışmada da, DMAH'ların halen en çok kullanılan ajan oldukları, ancak son üç yıl içinde cerrahların yaklaşık olarak üçte birinin kılavuzlardaki değişikliklere uyumlu olarak VTE profilaksisinde tercih değişimine gittikleri ve bundan dolayı, asetilsalisilik asit ve oral antikoagülan kullanımında artış olduğu bildirilmiştir. ${ }^{[61]}$ Yeterli düzeyde kanıta dayalı bilgi olmamasına rağmen elektif ya da travmatik nedenlerle yapılan ayak - ayak bileği cerrahisi sonrası çoğu hekim tedbir amaçlı VTE profilaksisi uygulamaktadır. Benzer şekilde, omuz artroplastisi sonrasında da VTE profilaksisi gerekliliği ve ideal ajan konusunda fikir birliği oluşmamıştır. [62]

DMAH'ler ve indirekt Faktör Xa inhibitörleri laboratuvar monitorizasyonu gerektirmez. Fakat bu ilaçların cilt altı enjeksiyon yoluyla kullanılması, bazı hastalarda uyum problemini beraberinde getirmektedir. VTE profilaksisinde güvenle ve etkin şekilde kullanılan bu ajanların istenmeyen kanama etkisine dair birçok randomize çalışma yapılmıştır. ${ }^{[23,63-66]}$ DMAH'ler ile warfarini karşılaştıran randomize çalışmalar, semptomatik VTE olaylarını engellemede DMAH'lerin warfarin kadar etkin, fakat kanama riskinin $\mathrm{DMAH}$ uygulamasında daha fazla olduğunu bildirmektedir. ${ }^{[63-65]}$

DMAH, fondaparinuks ve warfarine ilave olarak 2008'den beri, dabigatran, rivaroksaban, apiksaban ve edoksaban gibi birçok yeni nesil oral antikoagülan ajan onay alarak klinik kullanıma girmiştir. Bu ajanlar oral olarak uygulanmakta ve laboratuvar takibi gerektirmemektedir. Ancak, bu ajanların kullanımı ardından, ciddi ameliyat sonrası kanama riski mevcuttur. ${ }^{[21]}$ Olası bir kanama komplikasyonunda, dabigatran dışındaki yeni nesil oral antikoagülan ajanların etkisini nötralize edebilecek antidot yoktur. ${ }^{[10,21,40]}$

Yeni nesil oral antikoagülanlarla DMAH'leri karşılaştıran meta-analizler, TKA ve TDA sonrası VTE profilaksisi açısından yeni nesil oral antikoagülanların daha etkin olduğu sonucuna ulaşmıştır. ${ }^{[67,68]}$ Bunun yanı sıra bu ajanlar, yara iyileşmesinde gecikme, kanama komplikasyonları ve buna bağlı olarak enfeksiyon riskini arttırma açısından masum değildir ve dikkatle kullanılmalıdır. ${ }^{[1,67,68]}$

TKA ${ }^{[69,70]}$ ve TDA ${ }^{[71,72]}$ sonrası rivaroksaban kullanımının validasyonu için yapılmış dört tane çok merkezli ileriye dönük randomize Faz III ilaç çalışması vardır. Altı binin üzerinde TKA hastasında rivaroksaban $(10 \mathrm{mg}$ ) ile enoksoparin (40 mg) etkinliği karşılaştırılmış ve total VTE olayı sıklığını azaltmada rivaroksabanın daha etkin olduğu sonucuna varılmıştır. ${ }^{[70]}$ Fakat, semptomatik VTE ve majör kanama açısından iki ajan arasında fark saptanmamıştır. ${ }^{[70]}$ TDA sonrası profilakside rivaroksaban $(10 \mathrm{mg}$ ) ile enoksaparin (günde tek doz $40 \mathrm{mg}$ ) etkinliğini karşılaştıran çalışma sonucunda, total ve semptomatik VTE'nin önlenmesinde rivaroksaban üstün bulunmuştur. ${ }^{[72]}$ Rivaroksabanın (10 mg) enoksaparin (günde iki doz, $30 \mathrm{mg}$ ) ile karşılaştırıldığı başka bir çalışmada ise, total VTE'nin önlenmesi (semptomatik ve asemptomatik) ve mortalitenin azaltılmasında rivaroksabanın daha başarılı olduğu, ancak semptomatik VTE olayları ve kanama oranları açısından iki ajanın benzer olduğu bulunmuştur. ${ }^{[71]}$

Apiksaban, TKA ${ }^{[73]}$ ve TDA ${ }^{[74,75]}$ sonrası VTE profilaksisinde güvenlik ve etkinliği gösterilmiş bir diğer yeni nesil potent oral antikoagülandır. ADVANCE-3 çalışmasında elektif TKA yapılan hastalarda günde iki defa apiksaban 2,5 mg ile enoksaparin $40 \mathrm{mg}$ uygulamasının etkinliği kıyaslanmış, VTE olaylarını engelleme ve major kanama açısından iki ajan arasında fark bulunamamıştır. ${ }^{[73]}$ ADVANCE-1 çalışması TDA sonrası günde tek doz apiksaban 2,5 mg ile 2 doz enoksaparin 30 mg uygulamasını kıyaslamış ve semptomatik DVT ve PE açısından fark bulamamış, majör ve minör kanama riski açısından apiksabanın daha güvenilir olduğunu raporlamıştır. ${ }^{[75]}$ ADVANCE-2 çalışması da günde tek doz apiksaban 2,5 mg ile tek doz enoksaparin $40 \mathrm{mg}$ uygulamasını kıyaslamış, apiksaban grubunda major VTE daha az görülmekle birlikte, semptomatik DVT ve major kanama riski açısından iki ajan arasında fark bulamamıştır. ${ }^{[74]}$

Dabigatran'ın TKA ${ }^{[76,77]}$ ve TDA ${ }^{[78,79]}$ sonrası VTE profilaksisinde etkin olduğuna dair çalışmalar mevcuttur. TKA sonrası enoksaparin $40 \mathrm{mg}$ ya da dabigatran $150 \mathrm{mg}$ ya da $220 \mathrm{mg}$ ile profilaksi yapılan hastalarda DVT, PE ve major kanama açısından fark bulunamamıştır. TDA sonrası enoksaparin (günde $40 \mathrm{mg}$ tek doz ya da $30 \mathrm{mg}$ iki doz) yada dabigatran (günde $150 \mathrm{mg}$ tek doz yada $220 \mathrm{mg}$ tek doz) uygulaması ile DVT, PE ve major kanama açısından fark bulunamamıştır. ${ }^{[78]}$ Buna karşın Bloch ve ark., dabigatran kullanılan hastalarda, yatış süresince DMAH ve taburculukta asetilsalisilik asit kullanılan hastalara göre, yara yeri akıntısında artış ve hastanede yatış süresinde uzama olduğunu bildirmiştir. ${ }^{[80]}$

Bir başka derlemede fondaparinuks ve rivaroksabanın majör ortopedik cerrahi sonrası VTE profilaksisinde enoksoparine göre daha etkin olduğu fakat fondaparinuks kullanımında kanama ile ilgili komplikasyonların daha fazla görüldüğü bildirilmiştir. ${ }^{[81]}$ Apiksaban serilerinde enoksoparine göre kanama riskinin daha düşük olduğu bildirilmiştir. ${ }^{\left[{ }^{[1]}\right.}$

Asetilsalisilik asidin VTE profilaksisinde etkili bir ajan olduğunu gösteren çok sayıda çalışma mevcuttur. ${ }^{[82-91]}$ Bunların başında, 4.088 elektif TKA ve TDP hastasının yanı sıra, 13.356 kalça kırığı hastası ile yapılan kontrollü randomize Pulmonary Embolism Prevention (PEP) çalışması gelmektedir.[91] 
Bu çalışmada, $160 \mathrm{mg}$ asetilsalisilik asidin PE riskini ve azaltmada plaseboya göre daha etkin olduğu gösterilmiştir. Son yıllarda, özellikle Kuzey Amerika'da olmak üzere, asetilsalisilik asit profilaksisi uygulamasının popülerliği artmaktadır. ${ }^{[55]}$ TDA sonrası kullanılan VTE profilaksi ajanlarının geriye dönük olarak analiz edildiği 307 merkezin 93.840 hastasının kayıtlarının incelendiği bir çalışmada, asetilsalisilik asidin, warfarin ve diğer enjektabl ajanlara göre anlamlı derecede daha kısa hastane yatışı gerektirdiği tespit edilmiştir. ${ }^{[84]}$ Asetilsalisilik asit kullanımının, DMAH ya da warfarine kıyasla cerrahi sahada daha az majör kanamaya neden olduğu bildirilmiştir. ${ }^{[92]}$ Randomize çok merkezli bir çalışmada TKA sonrası profilakside 10 günlük DMAH tedavisini takiben 28 günlük asetilsalisilik asit kullanan hasta grubunda daha az kanama komplikasyonu ve DMAH grubuna göre benzer etkinlik görüldüğü bildirilmiştir. ${ }^{[93]}$ Asetilsalisilik asit ve warfarinin periprostetik eklem enfeksiyonu (PEE) açısından karşılaştırıldığı bir çalışmada, asetilsalisilik asit kullanan hastalarda PPE riskinin daha düşük olduğu tespit edilmiştir. ${ }^{94]}$ Markov, kohort maliyet analizi yapan bir güncel çalışmasında, öncesinde VTE öyküsü olmayan düşük risk grubundaki hastalarda asetilsalisilik asidin daha akılcı bir profilaksi metodu olduğunu göstermiştir. ${ }^{[95]}$ Artroplasti sonrası VTE profilaksisi için asetilsalisilik asit veya warfarin kullanımını karşılaştıran, 28.923 hastayı kapsayan bir çalışmada, asetilsalisilik asit kullanan hastalarda semptomatik PE olgu sayısının, hastane yatış süresinin ve yara yeri problemlerinin anlamlı ölçüde daha az görüldüğü bildirilmiştir.[96] Özetle, asetilsalisilik asit son yıllarda tüm kılavuzlar tarafından güçlü olarak desteklenen, oral kullanılabilen, monitörizasyon gerektirmeyen, kanama riski düşük ve ucuz bir seçenek olarak ön plana çıkmıştır.

Sonuç olarak, uygun VTE profilaksi ajanı, kanama ve VTE riski birlikte değerlendirilerek belirlenmelidir. VTE'nin farmakolojik profilaksisi için çok sayıda farmakolojik ajan bulunmaktadır. Her ajanın etkinliğinin, avantajlarının ve dezavantajlarının bilinmesi ve hastaya özel olarak profilaksi tercihinin belirlenmesi önemlidir.

\section{KAYNAKLAR}

1. Falck-Ytter Y, Francis CW, Johanson NA, Curley C, Dahl OE, Schulman S, Ortel TL, Pauker SG, Colwell CW Jr.. Prevention of VTE in orthopedic surgery patients: Antithrombotic Therapy and Prevention of Thrombosis, 9th ed: American College of Chest Physicians Evidence-Based Clinical Practice Guidelines. Chest 2012;141(2 Suppl):e278S-e325S. Crossref

2. Parvizi J, Huang R, Raphael IJ, Arnold WV, Rothman RH. Symptomatic pulmonary embolus after joint arthroplasty: stratification of risk factors. Clin Orthop Relat Res 2014;472(3):903-12. Crossref
3. Sheth NP, Lieberman JR, Della Valle CJ. DVT prophylaxis in total joint reconstruction. Orthop Clin North Am 2010;41(2):273-80. Crossref

4. Adams C, Anger KE, Greenwood BC, FanikosJ. Antithrombotic Pharmacotherapy (Sect. IX, Chap. 110). In: Irwin RS, Rippe JM, editors. Irwin \& Rippe's Intensive Care Medicine, 7th ed. Philadelphia PA: Williams \& Wilkins; 2012. pp.1224-42.

5. Johanson NA, Lachiewicz PF, Lieberman JR, Lotke PA, Parvizi J, Pellegrini V, Stringer TA, Tornetta P, Haralson RH, Watters WC. Prevention of symptomatic pulmonary embolism in patients undergoing total hip or knee arthroplasty. J Am Acad Orthop Surg 2009;17(3):183-96. Crossref

6. National Guideline Centre, Royal College of Physicians. Venous thromboembolisminover16s. Reducingtheriskofhospital-acquired deep vein thrombosis or pulmonary embolism. NICE guideline NG89 (volume 1). Methods, evidence and recommendations, 2018. Available at: https://mww.nice.org.uk/guidance/ng89/ evidence/full-guideline-volume-1-pdf-4787002765

7. Mont MA, Jacobs JJ, Boggio LN, Bozic KJ, Della Valle CJ, Goodman SB, Lewis CG, Yates AJ, Watters WC, Turkelson CM, Wies JL, Donnelly P, Patel N, Sluka P. Preventing venous thromboembolic disease in patients undergoing elective hip and knee arthroplasty. J Am Acad Orthop Surg 2011;19(12):768-76. Crossref

8. Lieberman JR, Hsu WK. Prevention of venous thromboembolic disease after total hip and knee arthroplasty. J Bone Joint Surg Am 2005;87(9):2097-112. Crossref

9. Harter K, Levine M, Henderson SO. Anticoagulation drug therapy: a review. West J Emerg Med 2015;16(1):11-7. Crossref

10. Alquwaizani M, Buckley L, Adams C, FanikosJ. Anticoagulants: A Review of the Pharmacology, Dosing, and Complications. Curr Emerg Hosp Med Rep 2013;1(2):83-97. Crossref

11. Douketis JD. Pharmacologic properties of the new oral anticoagulants: a clinician-oriented review with a focus on perioperative management. Curr Pharm Des 2010;16(31):3436-41. Crossref

12. Ageno W, Gallus AS, Wittkowsky A, Crowther M, Hylek EM, Palareti G. Oral anticoagulant therapy: Antithrombotic Therapy and Prevention of Thrombosis, 9th ed: American College of Chest Physicians Evidence-Based Clinical Practice Guidelines. Chest 2012;141(2 Suppl):e44S-e88S. Crossref

13. Wadelius M, Sorlin K, Wallerman O, Karlsson J, Yue QY, Magnusson PK, Wadelius C, Melhus H.. Warfarin sensitivity related to CYP2C9, CYP3A5, ABCB1(MDR1) and other factors. Pharmacogenomics J 2004;4(1):40-8. Crossref

14. Guyatt $\mathrm{GH}, \mathrm{Akl} E A$, Crowther $M$, Gutterman DD, Schuünemann HJ; American College of Chest Physicians Antithrombotic Therapy and Prevention of Thrombosis Panel.. Executive summary: Antithrombotic Therapy and Prevention of Thrombosis, 9th ed: American College of Chest Physicians Evidence-Based Clinical Practice Guidelines. Chest 2012;141(2 Suppl):7S-47S. Crossref

15. Tran HA, Chunilal SD, Harper PL, Tran H, Wood EM, Gallus AS. An update of consensus guidelines for warfarin reversal. Med J Aust 2013;198(4):198-9. Crossref

16. Dager WE. Warfarin for venous thromboembolism prophylaxis after elective hip or knee arthroplasty: exploring the evidence, guidelines, and challenges remaining. Ann Pharmacother 2012;46(1):79-88. Crossref

17. Weitz JI. Blood coagulation and anticoagulant, fibrinolytic, and antiplatelet drugs (Chap. 30). In: Brunton LL, Chabner BA, Knollmann BC, editors. Goodman \& Gilman's The Pharmacological Basis of Therapeutics. New York: McGrawHill Companies, Inc.; 2011. pp.849-77. 
18. Bussey H, Francis JL; The Heparin Consensus Group. Heparin overview and issues. Pharmacotherapy 2004;24(8 Pt 2):103S-7S. Crossref

19. White RH, Ginsberg JS. Low-molecular-weight heparins: are they all the same? BrJ Haematol 2003;121(1):12-20. Crossref

20. Gresham C, Levine M, Ruha AM. Case files of the Medical Toxicology Fellowship at Banner Good Samaritan Medical Center in Phoenix, AZ. a non-warfarin anticoagulant overdose. J Med Toxicol 2009;5(4):242-9. Crossref

21. Lieberman JR, Heckmann N. Venous Thromboembolism Prophylaxis in Total Hip Arthroplasty and Total Knee Arthroplasty Patients: From Guidelines to Practice. J Am Acad Orthop Surg 2017;25(12):789-98. Crossref

22. Douketis JD, Berger PB, Dunn AS, Jaffer AK, Spyropoulos AC, Becker RC, Ansell J. The perioperative management of antithrombotic therapy: American College of Chest Physicians Evidence-Based Clinical Practice Guidelines (8th Edition). Chest 2008;133(6 Suppl):299S-339S. Crossref

23. Parvizi J, Ceylan HH, Kucukdurmaz F, Merli G, Tuncay I, Beverland D. Venous Thromboembolism Following Hip and Knee Arthroplasty: The Role of Aspirin. J Bone Joint Surg Am 2017;99(11):961-72. Crossref

24. Crowther MA, Warkentin TE. Bleeding risk and the management ofbleeding complications in patients undergoing anticoagulant therapy: focus on new anticoagulant agents. Blood 2008;111(10):4871-9. Crossref

25. Hetzel GR, Sucker $C$. The heparins: all a nephrologist should know. Nephrol Dial Transplant 2005;20(10):2036-42. Crossref

26. Laryea J, Champagne B. Venous thromboembolism prophylaxis. Clin Colon Rectal Surg 2013;26(3):153-9. Crossref

27. Garcia DA, Baglin TP, Weitz JI, Samama MM. Parenteral anticoagulants: Antithrombotic Therapy and Prevention of Thrombosis, 9th ed: American College of Chest Physicians Evidence-Based Clinical Practice Guidelines. Chest 2012;141(2 Suppl):e24S-e43S. Crossref

28. Wolowacz SE, Roskell NS, Plumb JM, Caprini JA, Eriksson BI. Efficacy and safety of dabigatran etexilate for the prevention of venous thromboembolism following total hip or knee arthroplasty. A meta-analysis. Thromb Haemost 2009;101(1):77-85. Crossref

29. NICE. Apixaban for the prevention of venous thromboembolism after total hip or knee replacement in adults. National Institute for Health and Care Excellence, 2012. Available at: https:// www.nice.org.uk/Guidance/ta245

30. Turpie AG, Bauer KA, Eriksson BI, Lassen MR. Fondaparinux vs enoxaparin for the prevention of venous thromboembolism in major orthopedic surgery: a meta-analysis of 4 randomized double-blind studies. Arch Intern Med 2002;162(16):183340. Crossref

31. Eriksson BI, Lassen MR; PENTasaccharide in HIp-FRActure Surgery Plus (PENTHIFRA Plus) Investigators. Duration of prophylaxis against venous thromboembolism with fondaparinux after hip fracture surgery: a multicenter, randomized, placebo-controlled, double-blind study. Arch Intern Med 2003;163(11):1337-42. Crossref

32. Colwell CW Jr, Kwong LM, Turpie AG, Davidson BL. Flexibility in administration of fondaparinux for prevention of symptomatic venous thromboembolism in orthopaedic surgery. J Arthroplasty 2006;21(1):36-45. Crossref

33. Bijsterveld NR, Moons AH, Boekholdt SM, van Aken BE, Fennema H, Peters RJG, Meijers JCM, Büller HR, Levi M. Ability of recombinant factor VIla to reverse the anticoagulant effect of the pentasaccharide fondaparinux in healthy volunteers. Circulation 2002;106(20):2550-4. Crossref
34. Weitz JI, Eikelboom JW, Samama MM. New antithrombotic drugs: Antithrombotic Therapy and Prevention of Thrombosis, 9th ed: American College of Chest Physicians Evidence-Based Clinical Practice Guidelines. Chest 2012;141(2 Suppl):e120Se51S. Crossref

35. EINSTEIN Investigators, Bauersachs R, Berkowitz SD, Brenner B, Buller HR, Decousus H, Gallus AS, Lensing AW, Misselwitz F, Prins MH, Raskob GE, Segers A, Verhamme P, Wells P, Agnelli G, Bounameaux H, Cohen A, Davidson BL, Piovella F, Schellong S.. Oral rivaroxaban for symptomatic venous thromboembolism. N Engl J Med 2010;363(26):2499-510. Crossref

36. Kaatz S, Kouides PA, Garcia DA, Spyropolous AC, Crowther M, Douketis JD, Chan AKC, James A, Moll S, Ortel TL, Van Cott EM, Ansell J. Guidance on the emergent reversal of oral thrombin and factor Xa inhibitors. Am J Hematol 2012;87(Suppl 1):S141-5. Crossref

37. Yoshida WB, El Dib RP, de Alvarenga Yoshida R, de Abreu Maffei FH. Ximelagatran versus warfarin for prophylaxis of venous thromboembolism in major orthopedic surgery: systematic review of randomized controlled trials. Sao Paulo Med J 2006;124(6):355-61. Crossref

38. Ganetsky M, Babu KM, Salhanick SD, Brown RS, Boyer EW. Dabigatran: review of pharmacology and management of bleeding complications of this novel oral anticoagulant. J Med Toxicol 2011;7(4):281-7. Crossref

39. Eikelboom JW, Wallentin L, Connolly SJ, Ezekowitz M, Healey JS, Oldgren J, Yang S, Alings M, Kaatz S, Hohnloser SH, Diener HC, Franzosi MG, Huber K, Reilly P, Varrone J, Yusuf S.. Risk of bleeding with 2 doses of dabigatran compared with warfarin in older and younger patients with atrial fibrillation: an analysis of the randomized evaluation of long-term anticoagulant therapy (RE-LY) trial. Circulation 2011;123(21):2363-72. Crossref

40. Pollack CV Jr, Reilly PA, Eikelboom J, Glund S, Verhamme P, Bernstein RA, Dubiel R, Huisman MV, Hylek EM, Kamphuisen PW, Kreuzer J, Levy JH, Sellke FW, Stangier J, Steiner T, Wang B, Kam CW, Weitz JI.. Idarucizumab for Dabigatran Reversal. N Engl J Med 2015;373(6):511-20. Crossref

41. Wann LS, Curtis AB, Ellenbogen KA, Estes NA 3rd, Ezekowitz MD, Jackman WM, January CT, Lowe JE, Page RL, Slotwiner DJ, Stevenson WG, Tracy CM.. 2011 ACCF/AHA/ HRS focused update on the management of patients with atrial fibrillation (update on dabigatran): a report of the American College of Cardiology Foundation/American Heart Association Task Force on practice guidelines. J Am Coll Cardiol 2011;57(11):1330-7. Crossref

42. Fuster V, Sweeny JM. Aspirin: a historical and contemporary therapeutic overview. Circulation 2011;123(7):768-78. Crossref

43. Loll PJ, Picot D, Garavito RM. The structural basis of aspirin activity inferred from the crystal structure of inactivated prostaglandin H2 synthase. Nat Struct Biol 1995;2(8):63743. Crossref

44. Undas A, Brummel K, Musial J, Mann KG, Szczeklik A. Blood coagulation at the site of microvascular injury: effects of lowdose aspirin. Blood 2001;98(8):2423-31. Crossref

45. Undas A, Brummel-Ziedins K, Mann KG. Why does aspirin decrease the risk of venous thromboembolism? On old and novel antithrombotic effects of acetyl salicylic acid. J Thromb Haemost 2014;12(11):1776-87. Crossref

46. Eikelboom JW, Hirsh J, Spencer FA, Baglin TP, Weitz JI. Antiplatelet drugs: Antithrombotic Therapy and Prevention of Thrombosis, 9th ed: American College of Chest Physicians Evidence-Based Clinical Practice Guidelines. Chest 2012;141(2 Suppl):e89S-119S. Crossref 
47. Majerus PW. Arachidonate metabolism in vascular disorders. J Clin Invest 1983;72(5):1521-5. Crossref

48. Gurbel PA, Bliden KP, DiChiara J, Newcomer J, Weng W, Neerchal NK, Gesheff T, Chaganti SK, Etherington A, Tantry US.. Evaluation of dose-related effects of aspirin on platelet function: results from the Aspirin-Induced Platelet Effect (ASPECT) study. Circulation 2007;115(25):3156-64. Crossref

49. Villanueva GB, Allen N. Acetylation of antithrombin III by aspirin. Semin Thromb Hemost 1986;12(3):213-5. Crossref

50. Jesty J, Bluestein D. Acetylated prothrombin as a substrate in the measurement of the procoagulant activity of platelets: elimination of the feedback activation of platelets by thrombin. Anal Biochem 1999;272(1):64-70. Crossref

51. Valsami S, Ruf W, Leikauf MS, Madon J, Kaech A, Asmis LM. Immunomodulatory drugs increase endothelial tissue factor expression in vitro. Thromb Res 2011;127(3):264-71. Crossref

52. Svensson J, Bergman AC, Adamson U, Blomback M, Wallén $\mathrm{H}$, Jörneskog G. Acetylation and glycation of fibrinogen in vitro occur at specific lysine residues in a concentration dependent manner: a mass spectrometric and isotope labeling study. Biochem Biophys Res Commun 2012;421(2):335-42. Crossref

53. Undas A, Brummel K, Musial J, Mann KG, Szczeklik A. PI (A2) polymorphism of beta(3) integrins is associated with enhanced thrombin generation and impaired antithrombotic action of aspirin at the site of microvascular injury. Circulation 2001;104(22):2666-72. Crossref

54. Pedersen AK, FitzGerald GA. Dose-related kinetics of aspirin. Presystemic acetylation of platelet cyclooxygenase. N Engl J Med 1984;311(19):1206-11. Crossref

55. Azboy I, Barrack R, Thomas AM, Haddad FS, Parvizi J. Aspirin and the prevention of venous thromboembolism following total joint arthroplasty: commonly asked questions. Bone Joint J 2017;99-B(11):1420-30. Crossref

56. Parvizi J, Huang R, Restrepo C, Chen AF, Austin MS, Hozack WJ, Lonner JH. Low-Dose Aspirin Is Effective Chemoprophylaxis Against Clinically Important Venous Thromboembolism Following Total Joint Arthroplasty: A Preliminary Analysis. J Bone Joint Surg Am 2017;99(2):91-8. Crossref

57. Hirsh J, Guyatt G. Clinical experts or methodologists to write clinical guidelines? Lancet 2009;374(9686):273-5. Crossref

58. Markel DC, York S, Liston MJ Jr, Flynn JC, Barnes CL, Davis CM 3rd. Venous thromboembolism: management by American Association of Hip and Knee Surgeons. J Arthroplasty 2010;25(1):3-9.e2. Crossref

59. Groff H, Azboy I, Parvizi J. Differences in Reported Outcomes in Industry-Funded vs Nonfunded Studies Assessing Thromboprophylaxis After Total Joint Arthroplasty. J Arthroplasty 2018;33(11):3398-401. Crossref

60. Weisman MHS, Holmes JR, Irwin TA, Talusan PG. Venous Thromboembolic Prophylaxis in Foot and Ankle Surgery: A Review of Current Literature and Practice. Foot Ankle Spec 2017;10(4):343-51. Crossref

61. Azboy N, Cimen O, Demirtas A, Elci S, Azboy I. The changes in preferences for venous thromboembolism prophylaxis after total joint arthroplasty in Turkey: A survey. Eklem Hastalik Cerrahisi 2018;29(3):139-46. Crossref

62. Saleh HE, Pennings AL, ElMaraghy AW. Venous thromboembolism after shoulder arthroplasty: a systematic review. J Shoulder Elbow Surg 2013;22(10):1440-8. Crossref
63. Hull R, Raskob G, Pineo G, Rosenbloom D, Evans W, Mallory T, Anquist K, Smith F, Hughes G, Green D, Elliott CG, Panju A, Brant R.. A comparison of subcutaneous low-molecularweight heparin with warfarin sodium for prophylaxis against deep-vein thrombosis after hip or knee implantation. $N$ Engl J Med 1993;329(19):1370-6. Crossref

64. Colwell CW Jr, Collis DK, Paulson R, McCutchen JW, Bigler GT, Lutz S, Hardwick ME. Comparison of enoxaparin and warfarin for the prevention of venous thromboembolic disease after total hip arthroplasty. Evaluation during hospitalization and three months after discharge. J Bone Joint Surg Am 1999;81(7):932-40. Crossref

65. Hull RD, Pineo GF, Francis C, Bergqvist D, Fellenius C, Soderberg K, Holmqvist A, Mant M, Dear R, Baylis B, Mah A, Brant R.. Low-molecular-weight heparin prophylaxis using dalteparin in close proximity to surgery vs warfarin in hip arthroplasty patients: a double-blind, randomized comparison. The North American Fragmin Trial Investigators. Arch Intern Med 2000;160(14):2199-207. Crossref

66. Turpie AG, Bauer KA, Eriksson BI, Lassen MR. Postoperative fondaparinux versus postoperative enoxaparin for prevention of venous thromboembolism after elective hipreplacement surgery: a randomised double-blind trial. Lancet 2002;359(9319):1721-6. Crossref

67. Adam SS, McDuffie JR, Lachiewicz PF, Ortel TL, Williams JW Jr. Comparative effectiveness of new oral anticoagulants and standard thromboprophylaxis in patients having total hip or knee replacement: a systematic review. Ann Intern Med 2013;159(4):275-84. Crossref

68. Gomez-Outes A, Terleira-Fernandez Al, Suarez-Gea ML, Vargas-Castrillon E. Dabigatran, rivaroxaban, or apixaban versus enoxaparin for thromboprophylaxis after total hip or knee replacement: systematic review, meta-analysis, and indirect treatment comparisons. BMJ 2012;344:e3675. Crossref

69. Kakkar AK, Brenner B, Dahl OE, Eriksson BI, Mouret P, Muntz J, Soglian AG, Pap ÁF, Misselwitz F, Haas S. Extended duration rivaroxaban versus short-term enoxaparin for the prevention of venous thromboembolism after total hip arthroplasty: a double-blind, randomised controlled trial. Lancet 2008;372(9632):31-9. Crossref

70. Eriksson BI, Borris LC, Friedman RJ, Haas S, Huisman MV, Kakkar AK, Bandel TJ, Beckmann $H$, Muehlhofer E, Misselwitz F, Geerts W. Rivaroxaban versus enoxaparin for thromboprophylaxis after hip arthroplasty. N Engl J Med 2008;358(26):2765-75. Crossref

71. Turpie AG, Lassen MR, Davidson BL, Bauer KA, Gent M, Kwong LM, Cushner FD, Lotke PA, Berkowitz SD, Bandel TJ, Benson A, Misselwitz F, Fisher WD. Rivaroxaban versus enoxaparin for thromboprophylaxis after total knee arthroplasty (RECORD4): a randomised trial. Lancet 2009;373(9676):1673-80. Crossref

72. Lassen MR, Ageno W, Borris LC, Lieberman JR, Rosencher N, Bandel TJ, Misselwitz F, Turpie AGG. Rivaroxaban versus enoxaparin for thromboprophylaxis after total knee arthroplasty. N Engl J Med 2008;358(26):2776-86. Crossref

73. Lassen MR, Gallus A, Raskob GE, Pineo G, Chen D, Ramirez LM. Apixaban versus enoxaparin for thromboprophylaxis after hip replacement. N Engl J Med 2010;363(26):2487-98. Crossref

74. Lassen MR, Raskob GE, Gallus A, Pineo G, Chen D, Hornick $P$. Apixaban versus enoxaparin for thromboprophylaxis after knee replacement (ADVANCE-2): a randomised double-blind trial. Lancet 2010;375(9717):807-15. Crossref

75. Lassen MR, Raskob GE, Gallus A, Pineo G, Chen D, Portman $\mathrm{RJ}$. Apixaban or enoxaparin for thromboprophylaxis after knee replacement. N Engl J Med 2009;361(6):594-604. Crossref 
76. Eriksson BI, Dahl OE, Rosencher N, Kurth AA, van Dijk CN, Frostick SP, Prins MH, Hettiarachchi R, Hantel S, Schnee J, Büller HR. Dabigatran etexilate versus enoxaparin for prevention of venous thromboembolism after total hip replacement: a randomised, double-blind, non-inferiority trial. Lancet 2007;370(9591):949-56. Crossref

77. Eriksson $\mathrm{BI}$, Dahl OE, Huo $\mathrm{MH}$, Kurth $\mathrm{AA}$, Hantel $\mathrm{S}$, Hermansson K, Schnee JM, Friedman RJ; RE-NOVATE II Study Group.. Oral dabigatran versus enoxaparin for thromboprophylaxis after primary total hip arthroplasty (RENOVATE $\left.\|^{*}\right)$. A randomised, double-blind, non-inferiority trial. Thromb Haemost 2011;105(4):721-9. Crossref

78. Eriksson BI, Dahl OE, Rosencher N, Kurth AA, van Dijk CN, Frostick SP, Kälebo P, Christiansen AV, Hantel S, Hettiarachchi R, Schnee J, Büller HR; RE-MODEL Study Group.. Oral dabigatran etexilate vs. subcutaneous enoxaparin for the prevention of venous thromboembolism after total knee replacement: the RE-MODEL randomized trial. J Thromb Haemost 2007;5(11):2178-85. Crossref

79. RE-MOBILIZE Writing Committee, Ginsberg JS, Davidson BL, Comp PC, Francis CW, Friedman RJ, Huo MH, Lieberman JR, Muntz JE, Raskob GE, Clements ML, Hantel S, Schnee JM, Caprini JA.. Oral thrombin inhibitor dabigatran etexilate vs North American enoxaparin regimen for prevention of venous thromboembolism after knee arthroplasty surgery. J Arthroplasty 2009;24(1):1-9. Crossref

80. Bloch BV, Patel V, Best AJ. Thromboprophylaxis with dabigatran leads to an increased incidence of wound leakage and an increased length of stay after total joint replacement. Bone Joint J 2014;96-B(1):122-6. Crossref

81. de Alvarenga Yoshida R, Yoshida WB, de Abreu Maffei FH, El Dib R, Nunes R, Rollo HA. Systematic review of randomized controlled trials of new anticoagulants for venous thromboembolism prophylaxis in major orthopedic surgeries, compared with enoxaparin. Ann Vasc Surg 2013;27(3):35569. Crossref

82. Dorr LD, Gendelman V, Maheshwari AV, Boutary M, Wan Z, Long WT. Multimodal thromboprophylaxis for total hip and knee arthroplasty based on risk assessment. J Bone Joint Surg Am 2007;89(12):2648-57. Crossref

83. Nam D, Nunley RM, Johnson SR, Keeney JA, Barrack RL. Mobile compression devices and aspirin for VTE prophylaxis following simultaneous bilateral total knee arthroplasty. J Arthroplasty 2015;30(3):447-50. Crossref

84. Bozic KJ, Vail TP, Pekow PS, Maselli JH, Lindenauer PK, Auerbach AD. Does aspirin have a role in venous thromboembolism prophylaxis in total knee arthroplasty patients? J Arthroplasty 2010;25(7):1053-60. Crossref

85. Karthikeyan G, Eikelboom JW, Turpie AG, Hirsh J. Does acetyl salicylic acid (ASA) have a role in the prevention of venous thromboembolism? Br J Haematol 2009;146(2):142-9. Crossref
86. Lotke PA, Lonner JH. The benefit of aspirin chemoprophylaxis for thromboembolism after total knee arthroplasty. Clin Orthop Relat Res 2006;452:175-80. Crossref

87. McCardel BR, Lachiewicz PF, Jones K. Aspirin prophylaxis and surveillance of pulmonary embolism and deep vein thrombosis in total hip arthroplasty. J Arthroplasty 1990;5(2):181-5. Crossref

88. Huang RC, Parvizi J, Hozack WJ, Chen AF, Austin MS. Aspirin Is as Effective as and Safer Than Warfarin for Patients at Higher Risk of Venous Thromboembolism Undergoing Total Joint Arthroplasty. J Arthroplasty 2016;31(9 Suppl):83-6. Crossref

89. Ogonda L, Hill J, Doran E, Dennison J, Stevenson M, Beverland D. Aspirin for thromboprophylaxis after primary lower limb arthroplasty: early thromboembolic events and 90 day mortality in 11,459 patients. Bone Joint J 2016;98$B(3): 341-8$. Crossref

90. Deirmengian GK, Heller S, Smith EB, Maltenfort M, Chen AF, Parvizi J. Aspirin Can Be Used as Prophylaxis for Prevention of Venous Thromboembolism After Revision Hip and Knee Arthroplasty. J Arthroplasty 2016;31(10):2237-40. Crossref

91. Pulmonary Embolism Prevention (PEP) trial Collaborative Group. Prevention of pulmonary embolism and deep vein thrombosis with low dose aspirin: Pulmonary Embolism Prevention (PEP) trial. Lancet 2000;355(9212):1295-302. Crossref

92. Brown GA. Venous thromboembolism prophylaxis after major orthopaedic surgery: a pooled analysis of randomized controlled trials. J Arthroplasty 2009;24(6 Suppl):77-83. Crossref

93. Anderson DR, Dunbar MJ, Bohm ER, Belzile E, Kahn SR, Zukor D, Fisher W, Gofton W, Gross P, Pelet S, Crowther M, MacDonald S, Kim P, Pleasance S, Davis N, Andreou $\mathrm{P}$, Wells $\mathrm{P}$, Kovacs M, Rodger MA, Ramsay $T$, Carrier M, Vendittoli PA.. Aspirin versus low-molecular-weight heparin for extended venous thromboembolism prophylaxis after total hip arthroplasty: a randomized trial. Ann Intern Med 2013;158(11):800-6. Crossref

94. Huang R, Buckley PS, Scott B, Parvizi J, Purtill JJ. Administration of Aspirin as a Prophylaxis Agent Against Venous Thromboembolism Results in Lower Incidence of Periprosthetic Joint Infection. J Arthroplasty 2015;30(9 Suppl):39-41. Crossref

95. Mostafavi Tabatabaee R, Rasouli MR, Maltenfort MG, Parvizi J. Cost-effective prophylaxis against venous thromboembolism after total joint arthroplasty: warfarin versus aspirin. J Arthroplasty 2015;30(2):159-64. Crossref

96. Raphael IJ, Tischler EH, Huang R, Rothman RH, Hozack WJ, Parvizi J. Aspirin: an alternative for pulmonary embolism prophylaxis after arthroplasty? Clin Orthop Relat Res 2014;472(2):482-8. Crossref 